\title{
Posterior Vertebral Column Resection for Pediatric Congenital Kyphosis: A Case Report and Technical Concerns
}

\begin{abstract}
Junhyung Kim, Un Yong Choi, Kyung-Hyun Kim
Department of Neurosurgery, Gangnam Severance Hospital, Spine and Spinal Cord Institute, Yonsei University College of Medicine, Seoul, Republic of Korea

Corresponding author:

Kyung-Hyun Kim

Department of Neurosurgery,

Gangnam Severance Hospital, Spine and Spinal Cord Institute, Yonsei University College of Medicine, 211 Eonju-ro, Gangnam-gu, Seoul 06273, Republic of Korea

Tel: +82-2-2019-3390

Fax: +82-3-3461-9229

E-mail: nskhk@yuhs.ac

Treatment of severe congenital kyphosis in pediatric patients is challenging. Posterior vertebral column resection (PVCR) allows for correcting of severe deformities of the vertebral column, although it is technically demanding. A 12-year-old male who had been previously diagnosed with spine deformity due to the type 1 cervicothoracic junction kyphosis presented with progressive lower extremity paraparesis of motor grade 2 . On spine X-rays, the cervical lordosis was $70.3^{\circ}$, the thoracic kyphosis was $47.3^{\circ}$, and the lumbar lordosis was $-18.5^{\circ}$. To correct the patient's cervicothoracic kyphosis, we performed a PVCR of T1-T5, and posterior screw fixations of $\mathrm{C} 5-\mathrm{C} 7$ and T6-T8. Intraoperative monitoring was attempted, but did not provide detectable signals during the operation. Imaging studies on post-operative day 3 demonstrated cervical lordosis was $35.6^{\circ}$, and the Cobb angle of C7-T6 was $29.3^{\circ}$. The patient neurological status improved from category $C$ on the American Spinal Association Scale to category $D$, and was discharged without major complications. PVCR can offer several advantages such as providing larger correction angles and allowing both posterior and anterior manipulation of the spine from a single approach. Due to technical difficulties, a surgeon must thoroughly evaluate the surgical plan in order to avoid lethal complications.
\end{abstract}

Received: March 30, 2019

Revised: April 4, 2019

Accepted: April 13, 2019
Key Words: Congenital kyphosis; Pediatric deformity; Type 6 osteotomy; Vertebral column osteotomy

\section{INTRODUCTION}

Congenital kyphosis is a rare congenital deformity caused by developmental anomalies that impair longitudinal growth and vertebral rotation in the sagittal axis ${ }^{10)}$. It often causes uncontrolled pain and severe neurologic deficits in pediatric patients, which are frequently aggravated by the adolescent growth spurt.

Corrective surgery for congenital kyphosis involving upper thoracic region is challenging; intra-operative and post-operative complications include neurologic deterioration and mortality due to limitations such as invisible spine imaging on X-rays, proximal fixation points including cervical spine, and vulnerable spinal cord in this area. Thus, surgical procedures should be carefully planned to minimize risk. Posterior vertebral column resection (PVCR) technique has an advantage of allowing both anterior and posterior manipulations from a single approach $^{15)}$, but the reports regarding its application on pediatric patients are rare. We present a case of congenital kyphosis of the upper thoracic region treated with PVCR to share lessens we have learned.

\section{CASE REPORT}

\section{History and examination}

A 12-year-old male from Mongolia with no known medical history presented with progressive gait disturbance and kyphotic deformity of the cervicothoracic junction that had rapidly progressed in the last 2 years. At the time of the admission, the patient complained of severe upper back pain, and his neurologic status was determined to be category $\mathrm{C}$ on the American Spinal Injury Association Impairment Scale ${ }^{8)}$, he exhibited urinary urgency and constipation, and rigidity in both legs, causing him to be dependent on walker support during ambulation. On spine X-rays, cervical lordosis (determined by the $\mathrm{C} 2-\mathrm{C} 7$ Cobb angle) was measured at $70.3^{\circ}$, thoracic kyphosis (determined by the T1-T12 Cobb angle) was $47.3^{\circ}$, lumbar lordosis (determined by the L1-L5 Cobb angle) was $-18.5^{\circ}$, and the C7-T6 Cobb angle was $64.2^{\circ}$ (Fig. 1). Three-dimensional com- 
puted tomography revealed anterior and medial aplasia in the T2-T4 vertebrae and anterior hypoplasia in T1 and T5, indicating type 1 deformity. Also, the patient's pulmonary function was compromised due to pectus carinatum.

\section{PVCR}

The patient received PVCR of T1-2-3-4-5, posterior screw
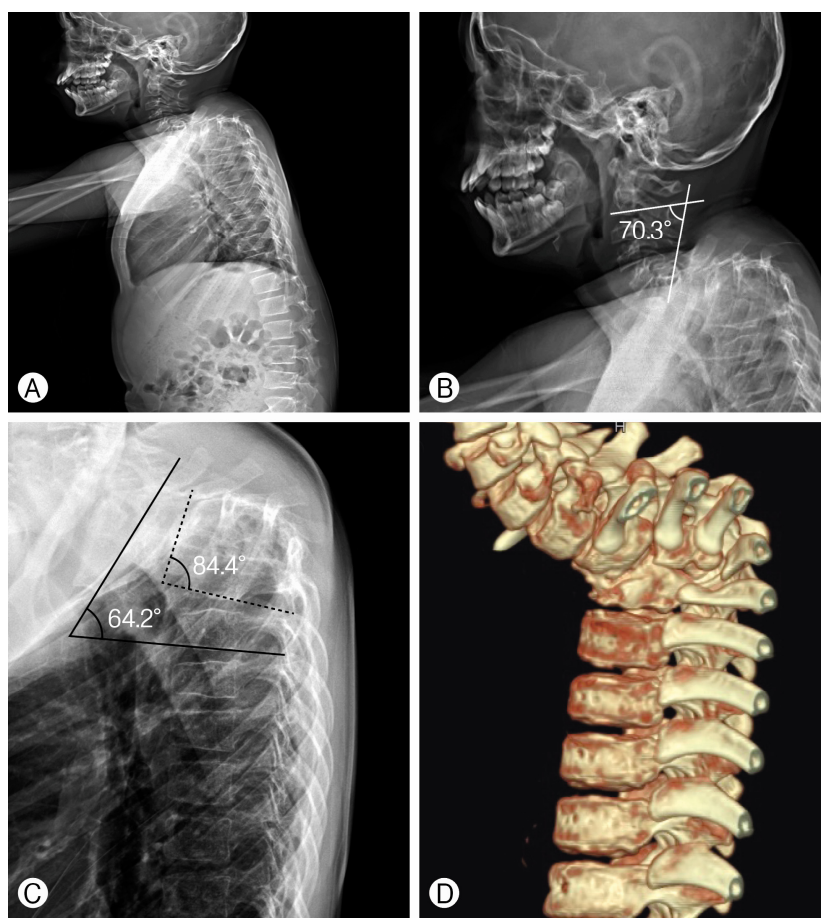

Fig. 1. Pre-operative X-ray images. (A, B) A whole spine lateral X-ray image showing severe kyphosis at the cervicothoracic junction and loss of lordosis of the lumbar spine. (C) C7-T6 Cobb angle is 64.2'. (D) Three-dimensional computed tomography scan demonstrating formation failure of the vertebral bodies of T2, T3, and T4. fixations of C5-6-7-T6-7-8, and posterolateral interbody fusion with autobone. As the operation began, trans-4-aminomethylcyclohexane-1-carboxylic acid (TXA) was applied to reduce intraoperative bleeding by immediately giving $500 \mathrm{mg}$ TXA intravenously followed by dripping a TXA solution at $5 \mathrm{mg} / \mathrm{kg} / \mathrm{h}$. After meticulous dissection, resection of the transverse processes and rib heads of T2-T5 was followed by laminectomies of T1, 2, 3, 4, and 5. Pedicle screws were inserted on each side of the vertebrae using the free-hand technique because intraoperative fluoroscopic guidance was not useful for defining the exact anatomy of the cervicothoracic junction. Temporary rods were assembled on the opposite side to provide structural stability during the resection. The vertebral body resection was performed from the lateral to the medial direction, after which the temporary rod was removed and placed on the other side. The resection was repeated on the opposite side. The midline bridging bone of the posterior part of the vertebral body just ventral to the spinal cord was removed with gentle traction of the spinal cord. Finally, the rods were placed on both sides, and the wound was closed. Total operation time was $529 \mathrm{~min}$, and blood loss totaled $2,700 \mathrm{~mL}$. Intra-operative neurophysiologic monitoring (IONM) with motor-evoked potentials (MEPs) was implemented, but we were unable to acquire signals from the lower extremities at any point in the operation.

The patient was admitted to the intensive care unit after surgery and was moved to the general ward the day after. A Foley catheter was removed a week after the operation so that he could self-void; the patient's weakness in the lower limbs improved considerably such that he was able to walk with an assist walker 2 weeks after surgery. There were notable improvements in radiologic parameters: cervical lordosis improved to $35.6^{\circ}$, and Cobb angle of C7-T6, $29.3^{\circ}$ (Fig. 2). Cervicothoracic X-rays were taken at a Mongolian hospital 75 days after the operation to show the instrumentations and the sagittal alignment of the spine were well maintained, with the cervical lordosis of $34.5^{\circ}$ and the C7-T6 Cobb angle of $28.5^{\circ}$ (Fig. 3). The patient was able to walk with a cane 7
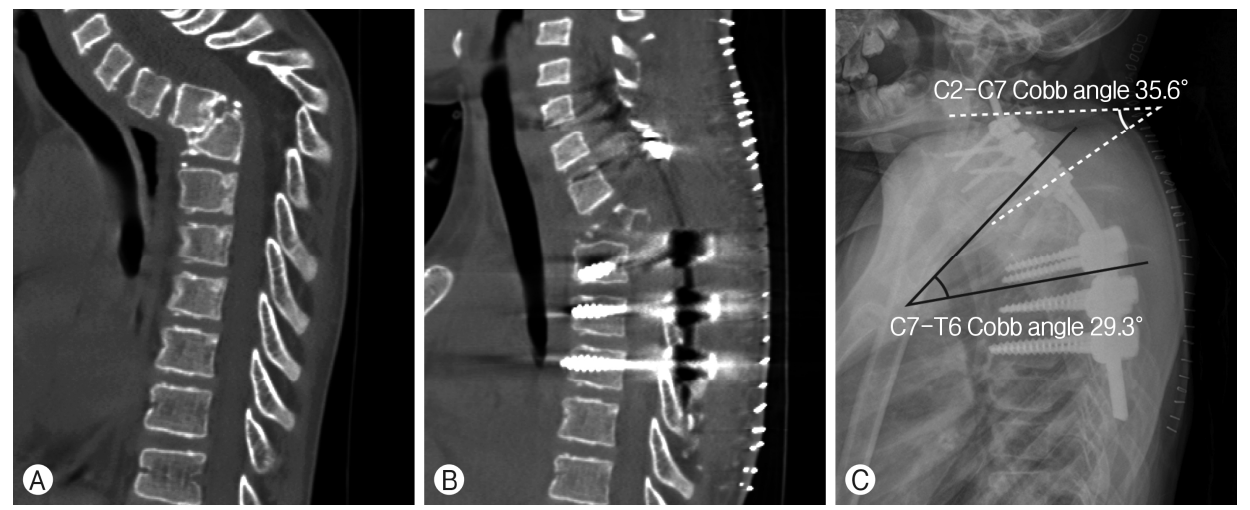

Fig. 2. Comparison of pre-operative and post-operative computed tomography (CT) images, and a postoperative X-ray image with improved sagittal parameters. (A) Pre-operative CT scan. (B) Post-operative CT scan. (C) Post-operative X-ray. The post-operative images were taken 3 days after the operation. 


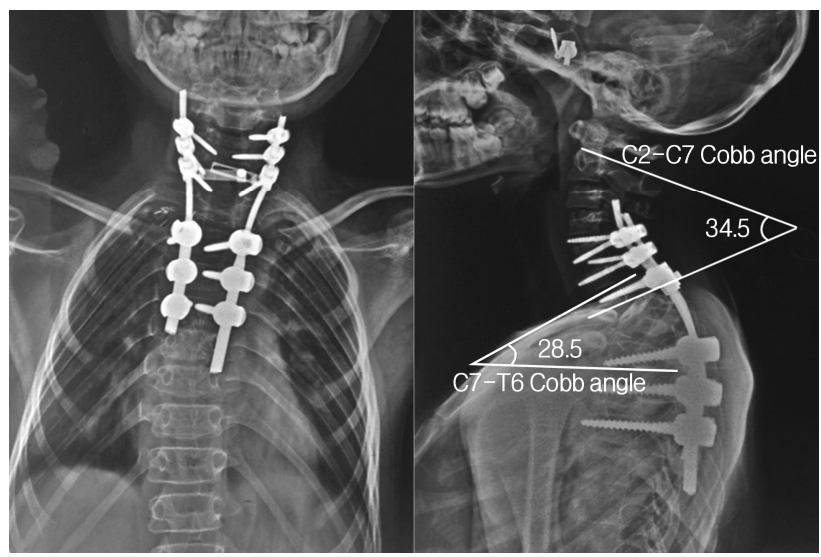

Fig. 3. Follow-up cenvicothoracic $X$-rays taken 75 days after the operation display the stability of the instrumentations and maintained sagittal alignment.

months after the operation.

\section{DISCUSSION}

Correction of a moderate spinal deformity can be effectively and safely performed from posterior, anterior, or posterior and anterior approaches ${ }^{1,5,13)}$. Winter and Hall ${ }^{18)}$ argued that severe deformity with thoracic kyphotic angle $>60^{\circ}$ and neurologic deficiency required surgical interventions from both anterior and posterior approaches to achieve adequate decompression and support of the structure. It is thought, however, that instrumentation without osteotomy may not allow sufficient correction for severe kyphosis; often, conventional osteotomy techniques such as Smith-Peterson osteotomy or pedicle subtraction osteotomy do not yield the desired correction. Moreover, a surgery requiring both anterior and posterior approach can be complicated by excessive intraoperative blood loss, a long operation time, and a manipulation of the thoracic cavity which increases a risk of respiratory complications. PVCR is a surgical method that can address these issues. In 2002, Suk et al. ${ }^{15)}$ introduced PVCR in an attempt to reduce the surgical complications and technical difficulties of VCR from simultaneous anterior and posterior approaches. Although it can cause lethal complications and is also technically challenging, PVCR offers several advantages. It allows both anterior and posterior column manipulation through a single posterior approach and yields a higher degree of deformity correction. In addition, PVCR helps preserve pulmonary function because the thoracic cavity is not involved. Ozturk et al. ${ }^{12)}$ reported an average Cobb angle correction rate of $62 \%$. Yang et al. ${ }^{20)}$ reported a mean correction of $58.9^{\circ}$ and a correction rate of $63.1 \%$ in patients with kyphosis. Using PVCR, we could correct the patient's severe kyphosis while reducing operation time and blood loss, without opening the thoracic cage.
In a spinal deformity correction surgery, massive intraoperative bleeding may occur as it frequently involves long level osteotomies and instrumentations. An extensive blood loss often necessitates transfusion, which can potentially cause postoperative complications such as pulmonary edema, hemolytic and non-hemolytic transfusion reactions ${ }^{17)}$, and wound infections ${ }^{6}$. Furthermore, the upper thoracic region is at a higher risk of spinal cord infarction during a spinal deformity surgery due to the inadequate collateral supply. Thus, during a corrective surgery for a spinal kyphosis, a surgeon must exploit all possible blood conservation strategies, one of which is the intra-operative use of TXA. There have been several studies advocating the efficacy of TXA in reducing intraoperative blood loss and the necessity of post-operative blood transfusion ${ }^{11,19)}$. Choi and his colleagues ${ }^{3)}$ argued that the use of TXA could effectively lower surgical bleeding, intra-operative transfusion requirements, and pos-toperative transfusion volume in patients receiving spinal deformity surgeries involving at least 5 vertebral levels. In addition to use of TXA, pre-operative intravenous ferric carboxymaltose infusion should be considered to reduce the need for blood transfusion and improve outcomes.

We encountered a few challenges during the operation. Although kyphosis in the upper thoracic spine with a kyphotic apex above $\mathrm{T} 5$ is uncommon, it is frequently accompanied by more serious neurological deficits ${ }^{9,10)}$. A complete removal of the midline bridging bone of the vertebral body required the utmost attention, as too much traction of the thoracic cord would cause neurologic impairments. For the same reason, hemostasis of the epidural bleeding was dangerous.

IONM is considered mandatory for any patient with congenital deformity undergoing a corrective surgery. There is a higher risk of post-operative paraplegia in patients with congenital spinal deformities than other causes of spinal deformities $^{9)}$. MEPs are known to be most sensitive to iatrogenic damages to the spinal cord and nerve roots ${ }^{14)}$, and their changes can occur due to hypotension, extreme manipulation of the cord, and osteotomy gap closing. However, in the presented case, intraoperative MEPs failed to detect any signal since the beginning of the surgery. We could find few publications that reported IONM failure in pediatric scoliosis patients with severe cerebral palsy ${ }^{4)}$, but the reasons for the monitoring failure remain to be further analyzed. We assume that IONM signals sometimes cannot be acquired in cases of severe deformity with neurologic deficits. One may consider awake surgery under local anesthesia as done in patients with multiple comorbidities if patients can tolerate ${ }^{2,7)}$. However, this may not always be feasible in pediatric patients who are less likely to cooperate well during the awake surgery.

Multilevel cervical fixation might be required in patients with kyphotic apex at upper thoracic spine, and it is of considerable difficulty especially in pediatric patients. In our case, we suspected that lateral mass of the patient was too small to endure corrective stress of screw fixation after surgery or 
the cantilever procedures during intra-operative correction, so we decided to insert cervical pedicle screws. An intra-operative C-arm X-ray device could not be utilized for the screw insertion in the cervicothoracic junction because of bone and soft tissue in the shoulder region. It is obviously more challenging to accurately insert screws in a deformed spine than in a spine with a normal anatomy. Intra-operative navigation using intraoperative computed scan or reformatted image of cervical and thoracic could be considered as one of the solutions, although it is not quite popular in our daily practice ${ }^{16)}$.

There are a few limitations to this case report. First of all, a longer follow-up period is necessary to evaluate the change of the patient's structural and neurologic status, as the bones are immature and ligaments are elastic during adolescent ages. We were able to confirm the neurologic improvements and radiological stability 7 months after the operation when we visited Mongolia, but a regular follow-up of the patient was practically impossible. Second, we need to collect and analyze more thoracic kyphosis cases to be able to address technical difficulties and solutions associated with PVCR in patients with congenital kyphosis.

Congenital kyphosis in the upper thoracic region may cause devastating consequences. PVCR is an effective surgical method for correcting severe kyphosis, allowing greater correction of the deformity angle, reduced operation time and blood loss. Since PVCR is a demanding procedure, surgeons must carefully analyze a case preoperatively and establish a detailed surgical plan.

\section{CONFLICTS OF INTEREST}

No potential conflict of interest relevant to this article was reported.

\section{REFERENCES}

1. Ayvaz M, Alanay A, Yazici M, Acaroglu E, Akalan N, Aksoy C: Safety and efficacy of posterior instrumentation for patients with congenital scoliosis and spinal dysraphism. J Pediatr Orthop 27:380-386, 2007

2. Chen HT, Tsai CH, Chao SC, Kao TH, Chen YJ, Hsu HC, et al.: Endoscopic discectomy of L5-S1 disc herniation via an interlaminar approach: Prospective controlled study under local and general anesthesia. Surg Neurol Int 2:93, 2011

3. Choi HY, Hyun SJ, Kim KJ, Jahng TA, Kim HJ: Effectiveness and safety of tranexamic acid in spinal deformity surgery. $\mathrm{J}$ Korean Neurosurg Soc 60:75-81, 2017

4. DiCindio S, Theroux M, Shah S, Miller F, Dabney K, Brislin RP, et al.: Multimodality monitoring of transcranial electric motor and somatosensory-evoked potentials during surgical correction of spinal deformity in patients with cerebral palsy and other neuromuscular disorders. Spine (Phila Pa 1976) 28:18511855, 2003

5. Hedequist DJ: Instrumentation and fusion for congenital spine deformities. Spine (Phila Pa 1976) 34:1783-1790, 2009

6. Janssen SJ, Braun Y, Wood KB, Cha TD, Schwab JH: Allogeneic blood transfusions and postoperative infections after lumbar spine surgery. Spine J 15:901-909, 2015

7. Khan MB, Kumar R, Enam SA: Thoracic and lumbar spinal surgery under local anesthesia for patients with multiple comorbidities: A consecutive case series. Surg Neurol Int 5:S62-S65, 2014

8. Kirshblum S, Waring W, 3rd: Updates for the international standards for neurological classification of spinal cord injury. Phys Med Rehabil Clin N Am 25:505-517, vii, 2014

9. MacEwen GD, Bunnell WP, Sriram K: Acute neurological complications in the treatment of scoliosis. A report of the Scoliosis Research Society. J Bone Joint Surg Am 57:404-408, 1975

10. McMaster MJ, Singh H: Natural history of congenital kyphosis and kyphoscoliosis. A study of one hundred and twelve patients. J Bone Joint Surg Am 81:1367-1383, 1999

11. Ng BKW, Chau WW, Hung AL, Hui AC, Lam TP, Cheng JC: Use of Tranexamic Acid (TXA) on reducing blood loss during scoliosis surgery in Chinese adolescents. Scoliosis 10:28, 2015

12. Ozturk C, Alanay A, Ganiyusufoglu K, Karadereler S, Ulusoy L, Hamzaoglu A: Short-term X-ray results of posterior vertebral column resection in severe congenital kyphosis, scoliosis, and kyphoscoliosis. Spine (Phila Pa 1976) 37:1054-1057, 2012

13. Ruf M, Jensen R, Letko L, Harms J: Hemivertebra resection and osteotomies in congenital spine deformity. Spine (Phila Pa 1976) 34:1791-1799, 2009

14. Schwartz DM, Auerbach JD, Dormans JP, Flynn J, Drummond DS, Bowe JA, et al.: Neurophysiological detection of impending spinal cord injury during scoliosis surgery. J Bone Joint Surg Am 89:2440-2449, 2007

15. Suk SI, Kim JH, Kim WJ, Lee SM, Chung ER, Nah KH: Posterior vertebral column resection for severe spinal deformities. Spine (Phila Pa 1976) 27:2374-2382, 2002

16. Tormenti MJ, Kostov DB, Gardner PA, Kanter AS, Spiro RM, Okonkwo DO: Intraoperative computed tomography imageguided navigation for posterior thoracolumbar spinal instrumentation in spinal deformity surgery. Neurosurg Focus 28:E11, 2010

17. Urban MK, Beckman J, Gordon M, Urquhart B, Boachie-Adjei O: The efficacy of antifibrinolytics in the reduction of blood loss during complex adult reconstructive spine surgery. Spine (Phila Pa 1976) 26:1152-1156, 2001

18. Winter RB, Hall JE: Kyphosis in childhood and adolescence. Spine (Phila Pa 1976) 3:285-308, 1978

19. Xie J, Lenke LG, Li T, Si Y, Zhao Z, Wang Y, et al.: Preliminary investigation of high-dose tranexamic acid for controlling intraoperative blood loss in patients undergoing spine correction surgery. Spine J 15:647-654, 2015

20. Yang C, Zheng Z, Liu H, Wang J, Kim YJ, Cho S: Posterior vertebral column resection in spinal deformity: a systematic review. Eur Spine J 25:2368-2375, 2016 